\title{
Separation of Mixed Signals in Dynamic Environments: Formulation and Some Implementation *
}

\author{
Ammar B.A. Gharbi \\ Fathi M. A. Salam \\ Systems, Circuits and Artificial Neural Networks Laboratory \\ Department of Electrical Engineering \\ Michigan State University, East Lansing, MI 48824 \\ email: gharbi@ee.msu.edu, salam@ee.msu.edu
}

\begin{abstract}
We describe algorithms and some chip implementation for separating unknown, but independent, mixture of temporal signals in static and dynamic environments. One algorithm is a modified HJ algorithm; the other is derived based on optimal control theory so as to maximize a functional which characterizes the linear independence of temporal signals. Moreover, we present some chip results and computer simulations and quantify the performance in static and dynamic environments.
\end{abstract}

\section{INTRODUCTION}

The Separation of Independent Sources (SIS) assumes that some unknown temporal signals propagate through a mixing and/or filtering medium. One is then interested in retrieving the original signals. A neural network may be built at the receiving end so as to counter the effect of the environment (see [3], [4], [5], [7], [8], [9].) (More motivation of the potential uses of this approach are relegated to the cited references due to space limitation.) Researchers working on the problem had assumed different models for the environment. We consider a linear dynamic model of the environment which in addition may account for dynamics generated by sensing devices, e.g. microphones, etc. Moreover, the approach permits direct extension of the model include nonlinearity for more general media. This paper considers the performance and chip implementation of a slight modification of the Herault-Jutten algorithm. It tests its performance in a static and dynamic media, and experimentally quantify its limitation. The paper also shows the existence of pa-

* Acknowledgment: This work is supported in part by ONR Grant N00014-92-J-1441 and the Michigan Research Excellence Fund (REF). rameters which would solve the dynamic case, i.e. retrieve the original signals from a mixed and filtered measurements. The paper includes an analytical formulation and an update law of the dynamic environment case using optimal control theory. It demonstrates partial results in this area via computer simulation. The paper is organized as follows. Section 2 defines the network architecture and the update law of the network parameters for two different models of media. In section 3, computer simulation of different medium models is performed. In section 4, the analysis of the performance of the modified HJ algorithm is tested in the presence of dynamics in the medium. Some concluding remarks are given in section 5 .

\section{ENVIRONMENT MODELING}

The general block diagram for SIS is shown in Figure 1 . The vectors $s(t), e(t)$, and $y(t)$ are respectively the unknown source vector, the received signal vector, and the output signal vector.

\subsection{Linear Static Medium}

The authors of [3], [4] and [5] proposed an algorithm that assumes a linear medium with no delays. The mixing matrix $\mathrm{A}$, whose diagonal entries are ones and which characterizes the medium, is assumed to be nonsingular. Specifically, the modified HJ algorithm is:

$$
\begin{aligned}
& e_{i}(t)=\sum_{i=1}^{n} a_{i j} s_{j}(t) \\
& \dot{y}_{i}(t)=-y_{i}(t)-\sum_{j \neq i} d_{i j} y_{j}(t)+e_{i}(t)
\end{aligned}
$$

Based on biological motivation, the heuristic update law proposed by Jutten is:

$$
\dot{d}_{i j}=\eta f\left(y_{i}\right) g\left(y_{j}\right)
$$


where $f($.$) and g($.$) are two nonlinear odd functions$ and $\eta$ is the learning rate. This algorithm has been implemented in CMOS and successful testing of its implementation using static network for equation (2) been reported in [1] and [2]. Moreover, simulation results (see Salam) have shown that system (1)-(3) successfully separation mixed signals more robustly. Our chip implementation of system (1)(3), discussed below, will be tested in both static and dynamic environments.

\subsection{Linear Dynamical Medium}

In [8], the problem of "blind" separation of independent signals in linear dynamic medium is presented. In this case, the input to the network model is no longer a linear combination of the unknown sources. Instead, it is a superimposed and a filtered ("delayed") version of the unknown sources. In [8], an algorithm is proposed. The environment and network models are specified as foolws:

$$
\begin{array}{ll}
\dot{\overline{\mathbf{x}}}=\bar{A} \overline{\mathbf{x}}+\overline{\mathbf{B}} \mathbf{~ s} & \dot{\mathbf{x}}=A \mathbf{x}+\mathbf{B} \mathbf{~ e} \\
\mathbf{e}=\overline{\mathbf{C}} \overline{\mathbf{x}}+\mathbf{D} \mathbf{~} \mathbf{y} & \mathbf{y}=\mathbf{C} \mathbf{x}+\mathbf{D} \mathbf{~}
\end{array}
$$

\subsubsection{A Theoretical Solution}

Suppose that the linear dynamical network $(A, B, C, D)$ converged to $\left(A^{*}, B^{*}, C^{*}, D^{*}\right)$ after successful learning. Then, by direct calculations,

$$
\begin{aligned}
A^{*} & =\bar{A}-\bar{B} \bar{D}^{-1} \bar{C} \\
B^{*} & =\bar{B} \bar{D}^{-1} \\
C^{*} & =-P \bar{D}^{-1} \bar{C} \\
D^{*} & =P \bar{D}^{-1}
\end{aligned}
$$

is a parameter solution that enables the separation of temporal signals, where $\mathrm{P}$ is a permutation matrix. This verifies the existence of parameters. The next step is to develop/define an update law that enable the parameters to converge such parameter set.

\subsubsection{The Update Law}

In [8], the following functional was used to characterize the independence of the output signal vector:

$$
P\left(\tau, t_{0}\right)=\int_{t_{0}}^{\tau} \mathbf{y}(t) \mathbf{y}^{T}(t) d t
$$

The scalar function:

$$
\left.h\left(\tau, t_{0}\right)=\mathbf{r}^{T} \tau\right) P\left(\tau, t_{0}\right) \mathbf{r}(\tau)
$$

may serve as an energy function. In $[8], h\left(\tau, t_{0}\right)$ is used to capture the notion of linear independence where the signals $\mathbf{r}(\tau)$ is sufficiently rich or random. This energy function is maximized in order to optimize the parameters of the network model. Using the continuous-time gradient method, the update law for the parameters are directly derived to be

$$
\begin{aligned}
\dot{A} & =\eta \int_{t_{0}}^{\tau} \lambda \mathbf{x}^{T} d t \\
\dot{B} & =\eta \int_{t_{0}}^{\tau} \lambda \mathbf{e}^{T} d t \\
\dot{C} & =\eta \int_{t_{0}}^{\tau} \mathbf{r y r}^{T} \mathbf{x}^{T} d t \\
\dot{D} & =\eta \int_{t_{0}}^{\tau} \mathbf{r y r}^{T} \mathbf{e}^{T} d t
\end{aligned}
$$

where, for simplicity, the same learning rate $\eta$ is used.

\section{COMPUTER SIMULATIONS}

Some computer simulations have been performed to check the validity of the algorithm in the case of static as well as dynamic mixing. A sample simulation for the proposed algorithm is shown in Figure 2 , where $\bar{A}=\left[\begin{array}{llll}-1 & 0.3 ; 0.4-1\end{array}\right], \bar{B}=\bar{C}=\left[\begin{array}{cccc}1 & 0 ; & 0 & 1\end{array}\right]$ and $\bar{D}=\left[\begin{array}{ll}1 & 0.8 ; 0.5\end{array}\right]$. More simulation scenario are presently being conducted. Once theoretical prediction are validated via computer simulation, we plan to implement the optimal control based learning algorithms into silicon.

\section{WHAT ABOUT DYNAMICS IN THE ENVIRONMENT?}

The modified HJ algorithm was implemented in VLSI using $2.0 \mu \mathrm{m}$ technology on a Tiny Chip $(2.22 \times$ $2.25 \mathrm{~mm}^{2}$.) We used similar building blocks to that of the Vittoz-Arreguit implementation. The basic components of the circuit are a transconductance amplifier [6] and sine hyperbolic [2]. Figures 34 shows the circuit diagrams of these basic components and their corresponding chip results. The algorithm is then tested to quantify its performance for static and dynamic medium modeling.

\subsection{Linear Static Modeling}

It is assumed here that the mixed signal is a linear combination of the unknown source as described in (1). Chip testing has been carried on using a two neuron network where it is desired to separate two independent signals. Figures 5-7 show respectively perfect separation of a sine and a triangular signals, a square and a triangular signals, and segments of two musics (Arabic and English) from two different speakers. A range of the values of the mixing parameters $a_{i j}$ where the separation was successful was determined experimentally. For the signal samples we used, the estimated $a_{i j}$ variation is within 0.0 and 0.8 . Figure 8 shows the failure of the separation when the parameters, $a_{i j}$ 's, are outside of this range. 


\subsection{Dynamic Modeling}

In this case, it is assumed that the input to the network is a superposition and a delayed version of the unknown sources.

$$
e_{i}(t)=s_{i}(t)+\sum_{j \neq i} a_{i j} s_{j}\left(t-\tau_{i j}\right)
$$

where $\tau_{i j} / \eta \ll 1$ in order to guarantee convergence of the parameters $d_{i j}$. The test was carried out successfully to separate two sine signals of different frequencies as shown in Figure 9. It is then tested to separate two mixed speech signals. It was found that the interval range over which the separation occurs had shrunk considerably compared to the case of linear static medium. It works only for $a_{i j}<0.4$.

\section{CONCLUSION}

We defined explicitly the algorithm for separation of signals in linear dynamic environment which can be extended to nonlinear environments. Initial computer simulations show some promise and identify specifics. Further simulations are planned prior to silicon implementation. We have tested an implementation of the slightly modified HJ algorithm and explored its validity range. As in the HJ algorithm, there are limits in coping with dynamic environment. The fact that sensors have their dynamics underlines the need for such consideration. Despite success in special cases, there still challenging problems ahead.

\section{REFERENCES}

[1] Marc H. Cohen and G. Andreou. Current-mode subthreshold mos implementation of heraultjutten autoadaptive network. IEEE Journal of Solid-State Circuits, 27(5):714-727, May 1992.

[2] Vittoz and X. Arreguit. Cmos integration of herault-jutten cells for separation of sources. Proceedings Workshop on Analog VLSI and Neural Systems, May 1989.

[3] J. Herault and C. Jutten. Space or time adaptive signal processing by neural network models. Neural Network for computing, AIP Conference Proceedings, pages 207-211, April 1986.

[4] J. Herault and C. Jutten. Blind separation of sources, part 1: An adaptive algorithm based on neuromimetic architecture. Signal Processing, 24:1-10, July 1991.

[5] J. Herault and C. Jutten. Blind separation of sources, part 2: Problem statement. Signal Processing, 24:11-20, July 1991.

[6] Carver Mead. Analog VLSI and Neural Systems. Prentice Hall, New York, 1989.

[7] J. C. Platt and F. Faggin. Networks for the separation sources that are superimposed and delayed. Advances in Neural Information Processing systems, 4, 1991.
[8] F. M. A. Salam. An adaptive network for blind separation of independent signals. International Symposium on Circuits and Systems, 1:431-434, May 1993.

[9] E. Sorouchyari. Blind separation of sources, part 3: Stability analysis. Signal Processing, 24:2129, July 1991.

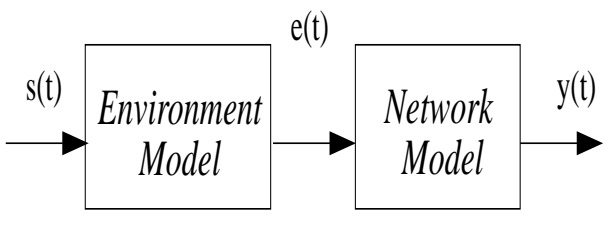

Figure 1. Architecture
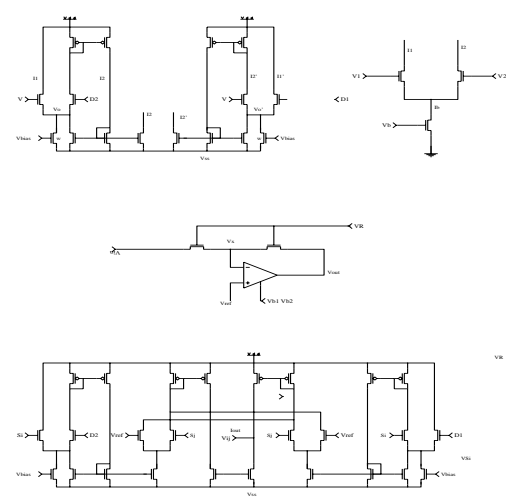

Figure 2. Analog components

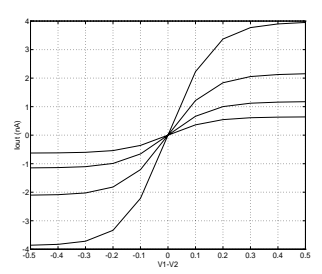

Hyperbolic tangent

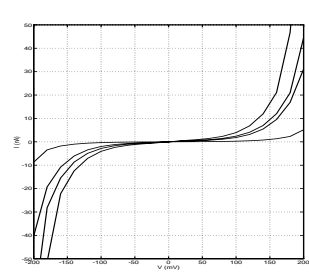

Hyperbolic sine
Figure 3. Basic Analog Components 

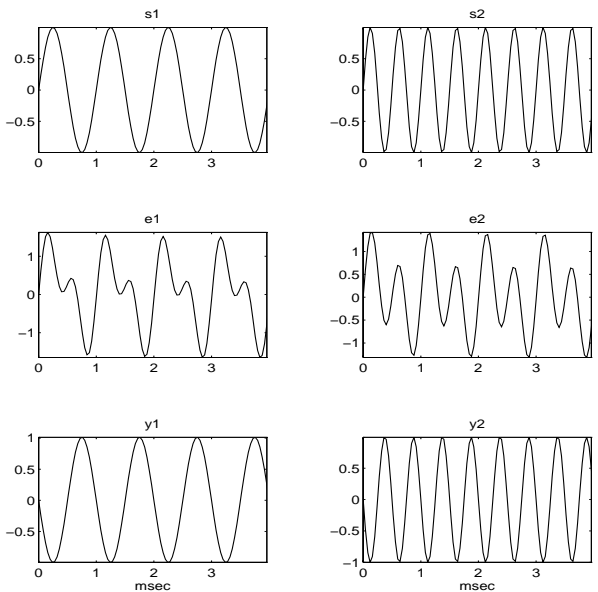

Figure 4. Dynamic Separation
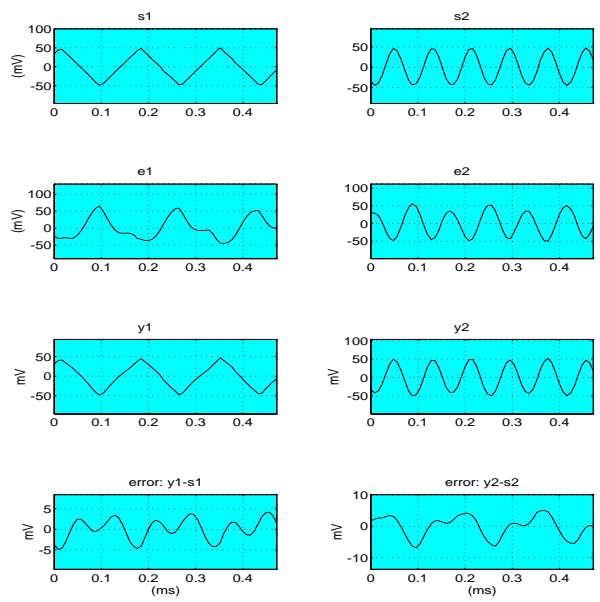

Figure 5. Static mixing: A sine and a triangular signals
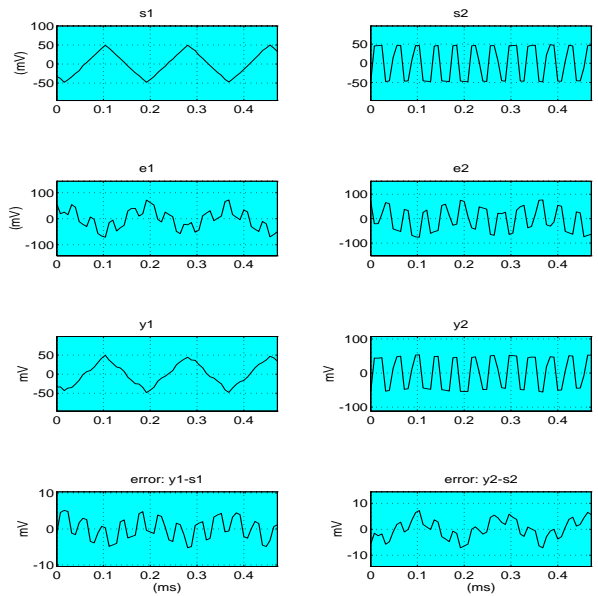

Figure 6. Static mixing: A square and a triangular signals
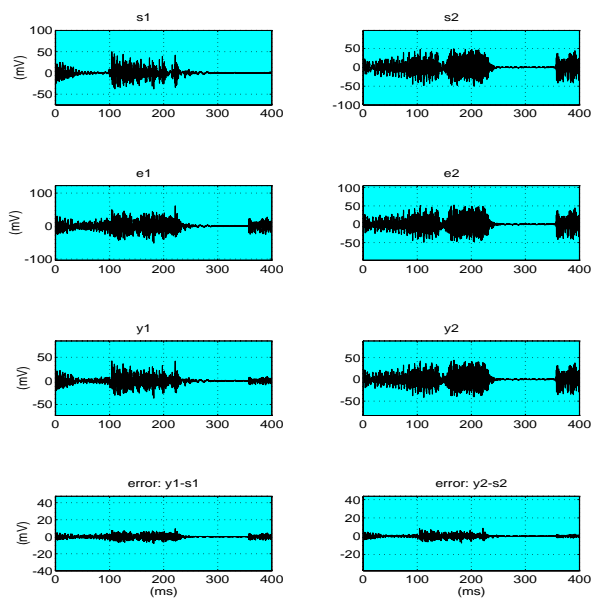

Figure 7. Static mixing: 2 speech signals
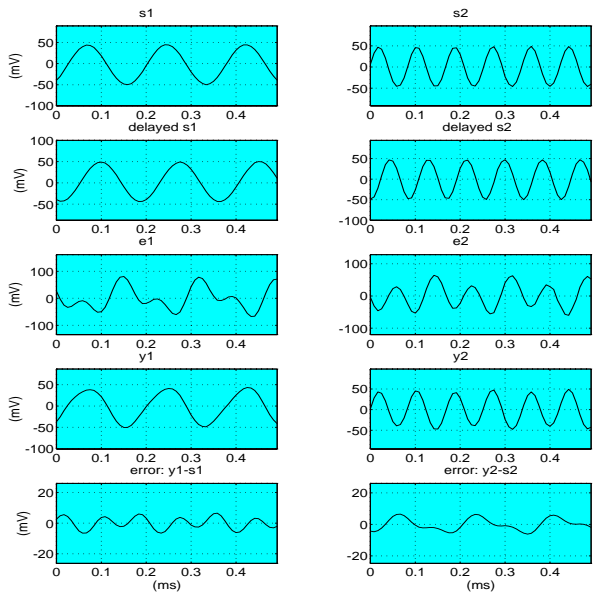

Figure 8. Dynamic mixing: 2 sine signals
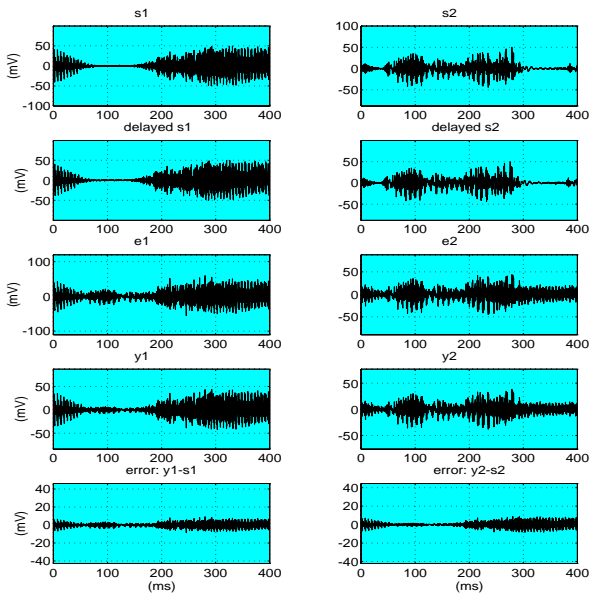

Figure 9. Dynamic mixing: 2 speech signals 